\title{
Exact Solutions and Symmetry Operators for the Nonlocal Gross-Pitaevskii Equation with Quadratic Potential
}

Alexander SHAPOVALOV ${ }^{\dagger \ddagger \S}$ Andrey TRIFONOV ${ }^{\ddagger}$ and Alexander LISOK $\S$

$\dagger$ Tomsk State University, 36 Lenin Ave., 634050 Tomsk, Russia

E-mail:shpv@phys.tsu.ru

$\ddagger$ Tomsk Polytechnic University, 30 Lenin Ave., 634050 Tomsk, Russia

E-mail: trifonov@mph.phtd.tpu.edu.ru

$\S$ Math. Phys. Laboratory, Tomsk Polytechnic University, 30 Lenin Ave., 634050 Tomsk, Russia

E-mail: lisok@mph.phtd.tpu.edu.ru

Received July 27, 2005, in final form October 06, 2005; Published online October 17, 2005

Original article is available at http://www.emis.de/journals/SIGMA/2005/Paper007/

\begin{abstract}
The complex WKB-Maslov method is used to consider an approach to the semiclassical integrability of the multidimensional Gross-Pitaevskii equation with an external field and nonlocal nonlinearity previously developed by the authors. Although the WKB-Maslov method is approximate in essence, it leads to exact solution of the GrossPitaevskii equation with an external and a nonlocal quadratic potential. For this equation, an exact solution of the Cauchy problem is constructed in the class of trajectory concentrated functions. A nonlinear evolution operator is found in explicit form and symmetry operators (mapping a solution of the equation into another solution) are obtained for the equation under consideration. General constructions are illustrated by examples.
\end{abstract}

Key words: WKB-Maslov complex germ method; semiclassical asymptotics; Gross-Pitaevskii equation; the Cauchy problem; nonlinear evolution operator; trajectory concentrated functions; symmetry operators

2000 Mathematics Subject Classification: 81Q20; 81Q30; 81R30

\section{Introduction}

Experimental advances in the realization of Bose-Einstein condensation (BEC) in weakly interacting alkali-metal atomic gases [1] have generated great interest in the theoretical study of the BEC. Its states and evolution are described using the Gross-Pitaevskii equation (GPE) [2, 3] for the wave function $\Psi(\vec{x}, t)$ of the condensate confined by external field with potential $V_{\text {ext }}(\vec{x}, t)$ at zero temperature:

$$
\left(-i \hbar \partial_{t}+\frac{\hat{\vec{p}}^{2}}{2 m}+V_{\mathrm{ext}}(\vec{x}, t)+\varkappa|\Psi(\vec{x}, t)|^{2}\right) \Psi(\vec{x}, t)=0 .
$$

Here $\vec{x} \in \mathbb{R}_{x}^{n}, \hat{\vec{p}}=-i \hbar \partial / \partial \vec{x}, t \in \mathbb{R}^{1}, \partial_{t}=\partial / \partial t,|\Psi|^{2}=\Psi^{*} \Psi, \Psi^{*}$ is complex conjugate to $\Psi, \varkappa$ is a real nonlinearity parameter, $|\Psi(\vec{x}, t)|^{2}$ is the condensate density, and $N[\Psi]=\int_{\mathbb{R}^{n}}|\Psi(\vec{x}, t)|^{2} d \vec{x}$ is the number of condensate particles. Following quantum mechanics, we refer to the solutions of the GPE as states.

Equation (1) is of nonlinear Schrödinger equation type with the local cubic nonlinearity $\varkappa|\Psi(\vec{x}, t)|^{2}$ representing the boson interaction in the mean field approximation. Besides the 
BEC, equation (1) describes a wide spectrum of nonlinear phenomena such as instability of water waves, nonlinear modulation of collisionless plasma waves, optical pulse propagation in nonlinear media and others. In all these cases, space localized soliton-like solutions are of principal interest. However, the wave packets described by equation (1) with $V_{\text {ext }}=0$ in multidimensional space $(n>1)$ with focusing nonlinearity $(\varkappa<0)$ are known to collapse, i.e. the situation where the wave amplitude increases extremely and becomes singular within a finite time or propagation distance (see, for example, [4] for reviews). To eliminate the collapse, which is considered as an artifact of the theory, one has to consider some effects that would render collapse impossible. The nonlocal form of nonlinearity is significant since it can, basically, eliminate collapse in all physical dimensions $(n=2,3)$ [5]. Meanwhile, in the derivation of the GPE (1), a nonlocal nonlinearity term $\int_{\mathbb{R}^{n}} V\left(\vec{x}, \vec{x}^{\prime}\right)\left|\Psi\left(\vec{x}^{\prime}, t\right)\right|^{2} d \vec{x}^{\prime} \Psi(\vec{x}, t)$ arises, and it is reduced to $\varkappa|\Psi(\vec{x}, t)|^{2} \Psi(\vec{x}, t)$, $\varkappa=\int V(\vec{x}) d \vec{x}$ if the potential $V\left(\vec{x}, \vec{x}^{\prime}\right)=V\left(\vec{x}-\vec{x}^{\prime}\right)$ is short-range [2].

In view of this consideration, of fundamental interest is the study of both the properties and the localized solutions of the nonlocal Gross-Pitaevskii equation. The integrability of the GPE is a nontrivial problem. In the one-dimensional case $(n=1)$ with $V_{\text {ext }}=0$, equation (1) (called the nonlinear Schrödinger equation (NLSE)) is known to be exactly integrable by the Inverse Scattering Transform (IST) method [6, 7]. This is the only IST integrable case since with $V_{\text {ext }} \neq 0$ the IST fails even in the one-dimensional case. The same is true for both the nonlocal GPE in all dimensions and the local GPE (1) in a multidimensional case $(n>1)$. Direct application of symmetry analysis $[8,9,10,11,12]$ to the nonlocal GPE is also hampered by presence of the nonlocal term and external potential in the equation.

In $[13,14,15,16]$ a semiclassical integrability approach was developed for a generalized nonlocal GPE named there a Hartree type equation:

$$
\begin{aligned}
& \left\{-i \hbar \partial_{t}+\hat{\mathcal{H}}_{\varkappa}(t)\right\} \Psi(\vec{x}, t)=\left\{-i \hbar \partial_{t}+\hat{\mathcal{H}}(t)+\varkappa \hat{V}(t, \Psi(t))\right\} \Psi(\vec{x}, t)=0, \\
& \Psi(\vec{x}, t) \in L_{2}\left(\mathbb{R}_{x}^{n}\right), \quad \hat{V}(t, \Psi(t))=\int_{\mathbb{R}^{n}} d \vec{y} \Psi^{*}(\vec{y}, t) V(\hat{z}, \hat{w}, t) \Psi(\vec{y}, t) .
\end{aligned}
$$

Here the linear operators $\hat{\mathcal{H}}(t)=\mathcal{H}(\hat{z}, t)$ and $V(\hat{z}, \hat{w}, t)$ are Weyl-ordered functions [17] of time $t$ and of noncommuting operators

$$
\hat{z}=(\hat{\vec{p}}, \vec{x})=(-i \hbar \partial / \partial \vec{x}, \vec{x}), \quad \hat{w}=(-i \hbar \partial / \partial \vec{y}, \vec{y}), \quad \vec{x}, \vec{y} \in \mathbb{R}^{n},
$$

with commutators

$$
\left[\hat{z}_{k}, \hat{z}_{j}\right]_{-}=\left[\hat{w}_{k}, \hat{w}_{j}\right]_{-}=i \hbar J_{k j}, \quad\left[\hat{z}_{k}, \hat{w}_{j}\right]_{-}=0, \quad k, j=\overline{1,2 n}
$$

$J=\left\|J_{k j}\right\|_{2 n \times 2 n}$ is a identity symplectic matrix $J=\left(\begin{array}{cc}0 & -\mathbb{I} \\ \mathbb{I} & 0\end{array}\right)_{2 n \times 2 n}, \mathbb{I}=\mathbb{I}_{n \times n}$ is an identity $(n \times n)$-matrix. This approach is based on the WKB-Maslov complex germ theory $[18,19]$ and gives a formal solution of the Cauchy problem, asymptotic in formal small parameter $\hbar(\hbar \rightarrow 0)$ accurate to $O\left(\hbar^{N / 2}\right)$, where $N$ is any natural number. The Cauchy problem was considered in the $\mathcal{P}_{\hbar}^{t}$ class of trajectory concentrated functions (TCFs) introduced in [13, 14]. Being approximate one in essence, the semiclassical approach results in some cases in exact solutions.

In the present work we construct an exact solution of the Cauchy problem in the $\mathcal{P}_{\hbar}^{t}$ class for equation (2) with the linear operators $\mathcal{H}(\hat{z}, t)$ and $V(\hat{z}, \hat{w}, t)$ being quadratic in $\hat{z}, \hat{w}$ :

$$
\begin{aligned}
& \mathcal{H}(\hat{z}, t)=\frac{1}{2}\left\langle\hat{z}, \mathcal{H}_{z z}(t) \hat{z}\right\rangle+\left\langle\mathcal{H}_{z}(t), \hat{z}\right\rangle \\
& V(\hat{z}, \hat{w}, t)=\frac{1}{2}\left\langle\hat{z}, W_{z z}(t) \hat{z}\right\rangle+\left\langle\hat{z}, W_{z w}(t) \hat{w}\right\rangle+\frac{1}{2}\left\langle\hat{w}, W_{w w}(t) \hat{w}\right\rangle .
\end{aligned}
$$


Here, $\mathcal{H}_{z z}(t), W_{z z}(t), W_{z w}(t), W_{w w}(t)$ are $2 n \times 2 n$ matrices, $\mathcal{H}_{z}(t)$ is a $2 n$-vector; $\langle\cdot, \cdot\rangle$ is an

Euclidean scalar product of vectors: $\langle\vec{p}, \vec{x}\rangle=\sum_{j=1}^{n} p_{j} x_{j} ; \vec{p}, \vec{x} \in \mathbb{R}^{n},\langle z, w\rangle=\sum_{j=1}^{2 n} z_{j} w_{j}, z, w \in \mathbb{R}^{2 n}$.

By solving the Cauchy problem, we obtain a nonlinear evolution operator in explicit form. With the evolution operator obtained, we formulate a nonlinear superposition principle for the solutions of the nonlocal GPE in the class of TCFs. Also, we give symmetry operators in general form that map each solution of the GPE into another solution. The general constructions are illustrated by examples.

\section{Cauchy problem}

Here we give a brief account of the solution of the Cauchy problem for equations (2), (3), (5), and (6), following $[13,14,15]$.

\section{The class of trajectory concentrated functions}

To set the Cauchy problem for equations (2), (3), (5), and (6), following [13, 14, 15], we define a class of functions $\mathcal{P}_{\hbar}^{t}$ via its generic element $\Phi(\vec{x}, t, \hbar)$ :

$$
\mathcal{P}_{\hbar}^{t}=\left\{\Phi: \Phi(\vec{x}, t, \hbar)=\varphi\left(\frac{\Delta \vec{x}}{\sqrt{\hbar}}, t, \hbar\right) \exp \left[\frac{i}{\hbar}(S(t, \hbar)+\langle\vec{P}(t, \hbar), \Delta \vec{x}\rangle)\right]\right\} .
$$

Here, the function $\varphi(\vec{\xi}, t, \hbar)$ belongs to the Schwartz space $\mathcal{S}\left(\mathbb{R}^{n}\right)$ with respect to $\vec{\xi} \in \mathbb{R}^{n}$, smoothly depends on $t$, and is regular in $\sqrt{\hbar}$ for $\hbar \rightarrow 0, \Delta \vec{x}=\vec{x}-\vec{X}(t, \hbar)$. The real function $S(t, \hbar)$ and the $2 n$-dimensional vector function $Z(t, \hbar)=(\vec{P}(t, \hbar), \vec{X}(t, \hbar))$ define the $\mathcal{P}_{\hbar}^{t}$ class, regularly depend on $\sqrt{\hbar}$ in the neighborhood of $\hbar=0$, and are to be determined. The functions of the $\mathcal{P}_{\hbar}^{t}$ class are normalizable with respect to the norm $\|\Phi(t)\|^{2}=\langle\Phi(t) \mid \Phi(t)\rangle$, where

$$
\langle\Psi(t) \mid \Phi(t)\rangle=\int_{\mathbb{R}^{n}} d \vec{x} \Psi^{*}(\vec{x}, t, \hbar) \Phi(\vec{x}, t, \hbar)
$$

is a scalar product in the space $L_{2}\left(\mathbb{R}_{x}^{n}\right)$. At any time $t \in \mathbb{R}^{1}$ the function $\Phi(\vec{x}, t, \hbar) \in \mathcal{P}_{\hbar}^{t}$ is localized in the limit $\hbar \rightarrow 0$ in the neighborhood of a point of the phase curve $z=Z(t, 0)$. For this reason we call $\mathcal{P}_{\hbar}^{t}$ the class of trajectory concentrated functions.

For $t=0$ the $\mathcal{P}_{\hbar}^{t}$ class transforms to the $\mathcal{P}_{\hbar}^{0}$ class of functions

$$
\begin{aligned}
& \psi(\vec{x}, \hbar)=\exp \left\{\frac{i}{\hbar}\left[S(0, \hbar)+\left\langle\vec{P}_{0}(\hbar),\left(\vec{x}-\vec{X}_{0}(\hbar)\right)\right\rangle\right]\right\} \varphi_{0}\left(\frac{\vec{x}-\vec{X}_{0}(\hbar)}{\sqrt{\hbar}}, \hbar\right), \\
& \varphi_{0}(\vec{\xi}, \hbar) \in \mathcal{S}\left(\mathbb{R}_{\xi}^{n}\right),
\end{aligned}
$$

where $Z_{0}(\hbar)=\left(\vec{P}_{0}(\hbar), \vec{X}_{0}(\hbar)\right)$ is a point of the phase space $\mathbb{R}_{p x}^{2 n}$, and the constant $S_{0}(\hbar)$ can be omitted without loss of generality. The Cauchy problem is formulated for equations (2), (3), (5), and (6) in the $\mathcal{P}_{\hbar}^{t}$ class of trajectory concentrated functions as

$$
\left.\Psi(\vec{x}, t, \hbar)\right|_{t=0}=\psi(\vec{x}, \hbar), \quad \psi \in \mathcal{P}_{\hbar}^{0}
$$

\section{Hamilton-Ehrenfest system}

To solve the Cauchy problem (2), (3), (5), (6), and (10), we first obtain the Hamilton-Ehrenfest system (HES) of equations in the moments of the solution $\Psi(\vec{x}, t, \hbar) \in \mathcal{P}_{\hbar}^{t}$ of equation (2). 
The operators $\mathcal{H}(\hat{z}, t)$, equation (5), and $V(\hat{z}, \hat{w}, t)$, equation (6), are self-adjoint, respectively, to the scalar product $(8)$ and to scalar product

$$
\langle\Psi(t) \mid \Phi(t)\rangle_{\mathbb{R}^{2 n}}=\int_{\mathbb{R}^{2 n}} d \vec{x} d \vec{y} \Psi^{*}(\vec{x}, \vec{y}, t, \hbar) \Phi(\vec{x}, \vec{y}, t, \hbar)
$$

in the space $L_{2}\left(\mathbb{R}_{x y}^{2 n}\right)$.

Define the mean value $\langle\hat{A}\rangle$ for a linear operator $\hat{A}(t)=A(\hat{z}, t)$ and a state $\Psi(\vec{x}, t, \hbar)$ as

$$
\langle\hat{A}\rangle=\frac{1}{\|\Psi(t)\|^{2}}\langle\Psi(t)|\hat{A}| \Psi(t)\rangle=A_{\Psi}(t, \hbar) .
$$

From (2), (3) we have

$$
\begin{aligned}
\frac{d}{d t}\langle\hat{A}(t)\rangle= & \left\langle\frac{\partial \hat{A}(t)}{\partial t}\right\rangle+\frac{i}{\hbar}\left\langle[\hat{\mathcal{H}}, \hat{A}(t)]_{-}\right\rangle \\
& +\frac{i \varkappa}{\hbar}\left\langle\int d \vec{y} \Psi^{*}(\vec{y}, t, \hbar)[V(\hat{z}, \hat{w}, t), \hat{A}(t)]_{-} \Psi(\vec{y}, t, \hbar)\right\rangle,
\end{aligned}
$$

where $[\hat{A}, \hat{B}]_{-}=\hat{A} \hat{B}-\hat{B} \hat{A}$ is the commutator of the operators $\hat{A}$ and $\hat{B}$. We refer to equation (12) as the Ehrenfest equation for the operator $\hat{A}$ and function $\Psi(\vec{x}, t, \hbar)$ as in quantum mechanics [20].

For $\hat{A}=1$, equation (12) gives $\|\Psi(t)\|^{2}=\|\Psi(0)\|^{2}=\|\Psi\|^{2}$. This implies that the norm of a solution of equations (2) and (3) does not depend on time, and we can use the parameter $\tilde{\varkappa}=\varkappa\|\Psi\|^{2}$ instead of $\varkappa$ in $(2)$.

Assume that

$$
\Delta_{\Psi \alpha}(t, \hbar)=\frac{\left\langle\Psi(t)\left|\{\Delta \hat{z}\}^{\alpha}\right| \Psi(t)\right\rangle}{\|\Psi\|^{2}}, \quad \alpha \in \mathbb{Z}_{+}^{2 n},
$$

are the moments of order $|\alpha|$ of the function $\Psi(\vec{x}, t)$ centered with respect to $z_{\Psi}(t, \hbar)=\left(\vec{p}_{\Psi}(t, \hbar)\right.$, $\left.\vec{x}_{\Psi}(t, \hbar)\right)$. Here $\{\Delta \hat{z}\}^{\alpha}$ is an operator with a Weyl symbol $\left(\Delta z_{\Psi}\right)^{\alpha}$,

$$
\Delta z_{\Psi}=z-z_{\Psi}(t, \hbar)=\left(\Delta \vec{p}_{\Psi}, \Delta \vec{x}_{\Psi}\right), \quad \Delta \vec{p}_{\Psi}=\vec{p}-\vec{p}_{\Psi}(t, \hbar), \quad \Delta \vec{x}_{\Psi}=\vec{x}-\vec{x}_{\Psi}(t, \hbar) .
$$

Along with (13) we use the following notation for the variances of coordinates, momenta, and correlations:

$$
\begin{aligned}
\Delta_{\Psi 2}(t, \hbar) & =\left\|\Delta_{\Psi j k}(t, \hbar)\right\|_{2 n \times 2 n}=\frac{1}{2\|\Psi\|^{2}}\left\|\left\langle\Psi(t)\left|\left\{\Delta \hat{z}_{j} \Delta \hat{z}_{k}+\Delta \hat{z}_{k} \Delta \hat{z}_{j}\right\}\right| \Psi(t)\right\rangle\right\|_{2 n \times 2 n} \\
& =\left(\begin{array}{cc}
\sigma_{p p}(t, \hbar) & \sigma_{p x}(t, \hbar) \\
\sigma_{x p}(t, \hbar) & \sigma_{x x}(t, \hbar)
\end{array}\right) \\
\sigma_{x p}(t, \hbar) & =\frac{1}{2}\left\|\left\langle\left\{\Delta x_{j} \Delta \hat{p}_{k}+\Delta \hat{p}_{k} \Delta x_{j}\right\}\right\rangle\right\|_{n \times n}, \\
\sigma_{x x}(t, \hbar) & =\left\|\left\langle\Delta x_{j} \Delta x_{k}\right\rangle\right\|_{n \times n}, \quad \sigma_{p p}(t, \hbar)=\left\|\left\langle\Delta \hat{p}_{j} \Delta \hat{p}_{k}\right\rangle\right\|_{n \times n} .
\end{aligned}
$$

The Ehrenfest equations (12) in mean values can be obtained for the operators $\hat{z}_{j},\{\Delta \hat{z}\}^{\alpha}$ and trajectory concentrated functions $(7)$ (see $[13,14]$ for details). However, for solving the equations under consideration, equations (2), (3), (5), and (6), we need only equations for the first-order and second-order moments

$$
\begin{aligned}
& \dot{z}_{\Psi}=J\left\{\mathcal{H}_{z}(t)+\left[\mathcal{H}_{z z}(t)+\tilde{\varkappa}\left(W_{z z}(t)+W_{z w}(t)\right)\right] z_{\Psi}\right\} \\
& \dot{\Delta}_{\Psi 2}=J\left[\mathcal{H}_{z z}(t)+\tilde{\varkappa} W_{z z}(t)\right] \Delta_{\Psi 2}-\Delta_{\Psi 2}\left[\mathcal{H}_{z z}(t)+\tilde{\varkappa} W_{z z}(t)\right] J
\end{aligned}
$$


We call the system (15) the Hamilton-Ehrenfest system (HES) of the second order for equations $(3),(5)$, and (6). Following [13, 14], we equate the functional vector-parameter $Z(t, \hbar)$ of the $\mathcal{P}_{\hbar}^{t}$ class of TCFs $(7)$ with $z_{\Psi}(t, \hbar)$, i.e. $\vec{p}_{\Psi}(t, \hbar)=\vec{P}(t, \hbar), \vec{x}_{\Psi}(t, \hbar)=\vec{X}(t, \hbar)$. This relates the ansatz (7) to an exact solution of equation (2).

Consider a phase space $\mathcal{M}^{N}$, $\operatorname{dim} \mathcal{M}^{N}=N=3 n+2 n^{2}$, of points $\mathfrak{g} \in \mathcal{M}^{N}$ with coordinates

$$
\begin{array}{ll}
\mathfrak{g}=(z, \Delta)^{\top}, & z \in \mathbb{R}^{2 n}, \quad z=(\vec{p}, \vec{x})^{\top}, \\
\Delta=\left(\Delta_{i j}\right)^{\top}, & \Delta_{i j}=\Delta_{j i}, \quad \Delta \in \mathbb{R}^{n+2 n^{2}}, \quad i, j=1, \ldots, 2 n .
\end{array}
$$

Here $A^{\top}$ is a matrix transposed to $A$. The coordinates of $\mathfrak{g} \in \mathcal{M}^{N}$ are written as matrix columns. The HES (15) can be considered a dynamic system in $\mathcal{M}^{N}$ :

$$
\begin{aligned}
& \dot{z}=J\left\{\mathcal{H}_{z}(t)+\left[\mathcal{H}_{z z}(t)+\tilde{\varkappa}\left(W_{z z}(t)+W_{z w}(t)\right)\right] z\right\}, \\
& \dot{\Delta}=J\left[\mathcal{H}_{z z}(t)+\tilde{\varkappa} W_{z z}(t)\right] \Delta-\Delta\left[\mathcal{H}_{z z}(t)+\tilde{\varkappa} W_{z z}(t)\right] J .
\end{aligned}
$$

With the substitution

$$
\Delta(t)=A(t) \Delta(0) A^{+}(t),
$$

equation (17) is rewritten in equivalent form:

$$
\dot{A}=J\left[\mathcal{H}_{z z}(t)+\tilde{\varkappa} W_{z z}(t)\right] A, \quad A(0)=\mathbb{I} .
$$

We call equation (18) a system in variations.

Denote by $\mathfrak{g}(t, \mathfrak{C})$ the general solution of equations (16), (17):

$$
\mathfrak{g}(t, \mathfrak{C})=\left(\vec{P}(t, \hbar, \mathfrak{C}), \vec{X}(t, \hbar, \mathfrak{C}), \Delta_{11}(t, \hbar, \mathfrak{C}), \Delta_{12}(t, \hbar, \mathfrak{C}), \ldots, \Delta_{2 n 2 n}(t, \hbar, \mathfrak{C})\right)^{\top}
$$

and by $\hat{\mathfrak{g}}$ - the operator column

$$
\hat{\mathfrak{g}}=\left(\hat{\vec{p}}, \vec{x},\left(\Delta \hat{p}_{1}\right)^{2}, \Delta \hat{p}_{1} \Delta \hat{p}_{2}, \ldots,\left(\Delta x_{n}\right)^{2}\right)^{\top} .
$$

Here

$$
\mathfrak{C}=\left(C_{1}, \ldots, C_{N}\right)^{\top} \in \mathbb{R}^{3 n+2 n^{2}}
$$

are arbitrary constants. Given constants $\mathfrak{C}$, equation (19) describes a trajectory of a point in the phase space $\mathcal{M}^{N}$.

Lemma 1. Let $\Psi(\vec{x}, t)$ be a partial solution of the GPE (2), (3), (5), (6) with an initial solution $\left.\Psi(\vec{x}, t)\right|_{t=0}=\psi(\vec{x})$. Define the constants $\mathfrak{C}(\Psi(t))$ by the condition

$$
\mathfrak{g}(t, \mathfrak{C})=\frac{1}{\|\Psi\|^{2}}\langle\Psi(t)|\hat{\mathfrak{g}}| \Psi(t)\rangle,
$$

and the constants $\mathfrak{C}(\psi)$ by the condition

$$
\mathfrak{g}(0, \mathfrak{C})=\frac{1}{\|\psi\|^{2}}\langle\psi|\hat{\mathfrak{g}}| \psi\rangle
$$

Then,

$$
\mathfrak{C}(\Psi(t))=\mathfrak{C}(\psi),
$$

i.e., $\mathfrak{C}(\Psi(t))$ are integrals of motion for equations (2), (3), (5), and (6). 
Proof. By construction, the vector

$$
\mathfrak{g}(t)=\frac{1}{\|\Psi\|^{2}}\langle\Psi(t)|\hat{\mathfrak{g}}| \Psi(t)\rangle=\mathfrak{g}(t, \mathfrak{C}(\Psi(t)))
$$

is a partial solution of the system $(16),(17)$, which coincides with $\mathfrak{g}(t, \mathfrak{C}(\psi))$ at the initial time $t=0$. In view of uniqueness of the solution of the Cauchy problem for the system (16) and (17), the equality

$$
\mathfrak{g}(t, \mathfrak{C}(\psi))=\mathfrak{g}(t, \mathfrak{C}(\Psi(t))),
$$

is valid. The proof is complete.

\section{Linear associated Schrödinger equation}

Let us substitute (5), (6) into (2), (3) and replace the operators $\hat{z}=(\hat{\vec{p}}, \vec{x})$ and $\hat{w}$ by $\Delta \hat{z}=$ $\hat{z}-z(t, \hbar, \mathfrak{C})=(\Delta \hat{\vec{p}}, \Delta \vec{x})=(\hat{\vec{p}}-\vec{P}(t, \hbar, \mathfrak{C}), \vec{x}-\vec{X}(t, \hbar, \mathfrak{C}))$ and $\Delta \hat{w}=\hat{w}-z(t, \hbar, \mathfrak{C})$. Then we have

$$
\begin{aligned}
&\left\{-i \hbar \partial_{t}+\hat{\mathfrak{H}}(t, \Psi(t))\right\} \Psi=0, \hat{\mathfrak{H}}(t, \Psi(t))= \\
& \mathfrak{H}(t, \Psi(t))+\left\langle\mathfrak{H}_{z}(t, \Psi(t)), \Delta \hat{z}\right\rangle+\frac{1}{2}\left\langle\Delta \hat{z}, \mathfrak{H}_{z z}(t, \Psi(t)) \Delta \hat{z}\right\rangle, \\
& \mathfrak{H}(t, \Psi(t))= \frac{1}{2}\left\langle z_{\Psi}(t, \hbar),\left[\mathcal{H}_{z z}(t)+\tilde{\varkappa}\left(W_{z z}(t)+2 W_{z w}(t)+W_{w w}(t)\right)\right] z_{\Psi}(t, \hbar)\right\rangle \\
&+\left\langle\mathcal{H}_{z}(t), z_{\Psi}(t, \hbar)\right\rangle+\frac{1}{2} \tilde{\varkappa} \mathrm{Sp}\left(W_{w w}(t) \Delta_{\Psi 2}\right), \\
& \mathfrak{H}_{z}(t, \Psi(t))= \mathcal{H}_{z}(t)+\left(\mathcal{H}_{z z}(t)+\tilde{\varkappa} W_{z z}(t)+\tilde{\varkappa} W_{z w}(t)\right) z_{\Psi}(t, \hbar), \\
& \mathfrak{H}_{z z}(t, \Psi(t))= \mathcal{H}_{z z}(t)+\tilde{\varkappa} W_{z z}(t) .
\end{aligned}
$$

Let us replace the mean values $z_{\Psi}(t, \hbar), \Delta_{\psi}(t, \hbar)$ in the nonlinear GPE (28) by the respective terms of the general solution (19) of the system (16), (17). As a result, we obtain a linear equation:

$$
\begin{aligned}
& \left\{-i \hbar \partial_{t}+\hat{\mathfrak{H}}(t, \mathfrak{g}(t, \mathfrak{C}))\right\} \Phi(\vec{x}, t, \hbar, \mathfrak{C})=0 \\
& \hat{\mathfrak{H}}(t, \mathfrak{g}(t, \mathfrak{C}))=\mathfrak{H}(t, \hbar, \mathfrak{C})+\left\langle\mathfrak{H}_{z}(t, \hbar, \mathfrak{C}), \Delta \hat{z}\right\rangle+\frac{1}{2}\left\langle\Delta \hat{z}, \mathfrak{H}_{z z}(t) \Delta \hat{z}\right\rangle, \\
& \mathfrak{H}(t, \hbar, \mathfrak{C})=\frac{1}{2}\left\langle z(t, \hbar, \mathfrak{C}),\left[\mathcal{H}_{z z}(t)+\tilde{\varkappa}\left(W_{z z}(t)+2 W_{z w}(t)+W_{w w}(t)\right)\right] z(t, \hbar, \mathfrak{C})\right\rangle \\
& +\left\langle\mathcal{H}_{z}(t), z(t, \hbar, \mathfrak{C})\right\rangle+\frac{1}{2} \tilde{\varkappa} \operatorname{Sp}\left(W_{z z}(t) \Delta(t, \hbar, \mathfrak{C})\right), \\
& \mathfrak{H}_{z}(t, \hbar, \mathfrak{C})=\mathcal{H}_{z}(t)+\left(\mathcal{H}_{z z}(t)+\tilde{\varkappa} W_{z z}(t)+\tilde{\varkappa} W_{z w}(t)\right) z(t, \hbar, \mathfrak{C}), \\
& \mathfrak{H}_{z z}(t)=\mathcal{H}_{z z}(t)+\tilde{\varkappa} W_{z z}(t), \quad z(t, \hbar, \mathfrak{C})=(\vec{P}(t, \hbar, \mathfrak{C}), \vec{X}(t, \hbar, \mathfrak{C})) .
\end{aligned}
$$

We call equation (31) the linear associated Schrödinger equation (LASE) for the nonlinear GrossPitaevskii equation (28). More precisely, equation (31) is to be considered as a family of equations parametrized by the constants $\mathfrak{C}$ of the form (21). Each element of the family (31) is a linear Schrödinger equation with a quadratic Hamiltonian with respect to operators of coordinates and momenta. Such an equation is well known to be solvable in explicit form (see, for example, [21, 22]). In particular, partial solutions can be found as Gaussian wave packets and a Fock basis of solutions, and Green function can be constructed. 


\section{LASE solutions and GPE solutions}

Consider a relationship between the solutions of the LASE and GPE. Let $\Phi(\vec{x}, t, \hbar, \mathfrak{C})$ be the solution of the Cauchy problem for the LASE (31)

$$
\left.\Phi(\vec{x}, t, \hbar, \mathfrak{C})\right|_{t=0}=\psi(\vec{x}, \hbar), \quad \psi \in \mathcal{P}_{\hbar}^{0} .
$$

The function $\Phi(\vec{x}, t, \hbar, \mathfrak{C})$ depends on arbitrary parameters $\mathfrak{C}$ which appear in the LASE (31). Let $\mathfrak{C}$ are subject to the condition $(23)$ and are functionals $\mathfrak{C}(\psi)$.

Theorem 1. The solution of the Cauchy problem (10) for the GPE (2) is

$$
\Psi(\vec{x}, t, \hbar)=\Phi(\vec{x}, t, \hbar, \mathfrak{C}(\psi)) .
$$

Proof. The function $\Phi(\vec{x}, t, \hbar, \mathfrak{C}(\psi))$ satisfies equation (31) for arbitrary $\mathfrak{C}$ and also for $\mathfrak{C}=\mathfrak{C}(\psi)$. According to equation (24), in Lemma 1 we do not violate the equality in (31) if we replace $\mathfrak{C}(\psi)$ by $\mathfrak{C}(\Psi(t))$. In view of equations $(25),(26)$, one can see that $\Phi(\vec{x}, t, \hbar, \mathfrak{C}(\psi))=\Phi(\vec{x}, t, \hbar, \mathfrak{C}(\Psi(t)))$ and the operator $\hat{\mathfrak{H}}(t, \mathfrak{g}(t, \mathfrak{C}))$ in $(31)$ becomes to $\hat{\mathfrak{H}}(t, \Psi(t))$ in $(27)$. This implies that the function $\Phi(\vec{x}, t, \hbar, \mathfrak{C}(\psi))$ satisfies equation $(27)$ and the initial condition (33), which correlates with (10). Consequently, equation (34) is valid, and the theorem is proved.

The relationship between the steps described above can be shown diagrammatically:



Theorem 1 makes it possible to obtain a nonlinear evolution operator for the GPE (27) in the $\mathcal{P}_{\hbar}^{t}$ class of TCFs (7). The evolution operator can be written as a nonlinear integral operator using the Green function of the LASE (31) with constants $\mathfrak{C}$ changed by $\mathfrak{C}(\psi)$ according to relation (34).

\section{Nonlinear evolution operator}

The Green function $G_{\varkappa}(\vec{x}, \vec{y}, t, s, \mathfrak{g}(t, \mathfrak{C}), \mathfrak{g}(s, \mathfrak{C}))$ for the Cauchy problem (31), (33) is defined by the conditions

$$
\begin{aligned}
& {\left[-i \hbar \partial_{t}+\hat{\mathfrak{H}}(t, \mathfrak{g}(t, \mathfrak{C}))\right] G_{\varkappa}(\vec{x}, \vec{y}, t, s, \mathfrak{g}(t, \mathfrak{C}), \mathfrak{g}(s, \mathfrak{C}))=0,} \\
& \lim _{t \rightarrow s} G_{\varkappa}(\vec{x}, \vec{y}, t, s, \mathfrak{g}(t, \mathfrak{C}), \mathfrak{g}(s, \mathfrak{C}))=\delta(\vec{x}-\vec{y}) .
\end{aligned}
$$

Here the operator $\hat{\mathfrak{H}}(t, \mathfrak{g}(t, \mathfrak{C}))$, given by (32), is quadratic in coordinates and momenta.

We shall seek for the required Green function under the simplifying assumption

$$
\operatorname{det} \mathfrak{H}_{p p}(s) \neq 0 \text {. }
$$

Following, for example, [21, 22], we obtain

$$
G_{\varkappa}(\vec{x}, \vec{y}, t, s, \mathfrak{g}(t, \mathfrak{C}), \mathfrak{g}(s, \mathfrak{C}))=\frac{1}{\sqrt{\operatorname{det}\left(-i 2 \pi \hbar \lambda_{3}(t, s)\right)}}
$$




$$
\begin{aligned}
& \times \exp \left\{\frac{i}{\hbar}[S(t, \hbar, \mathfrak{g}(t, \mathfrak{C}))-S(s, \hbar, \mathfrak{g}(s, \mathfrak{C}))+\langle\vec{P}(t, \hbar, \mathfrak{C}), \Delta \vec{x}\rangle-\langle\vec{P}(s, \hbar, \mathfrak{C}), \Delta \vec{y}\rangle\right. \\
& \left.\left.-\frac{1}{2}\left\langle\Delta \vec{y}, \lambda_{1}(t, s) \lambda_{3}^{-1}(t, s) \Delta \vec{y}\right\rangle+\left\langle\Delta \vec{x}, \lambda_{3}^{-1}(t, s) \Delta \vec{y}\right\rangle-\frac{1}{2}\left\langle\Delta \vec{x}, \lambda_{3}^{-1}(t, s) \lambda_{4}(t, s) \Delta \vec{x}\right\rangle\right]\right\} .
\end{aligned}
$$

Here, $\Delta \vec{y}=\vec{y}-\vec{X}(s, \hbar, \mathfrak{C}), n \times n$ matrices $\lambda_{k}(t, s), k=\overline{1,4}$, are blocks of the block matrix $\mathcal{A}(t, s)$ of the system in variations:

$$
\begin{aligned}
& \dot{\mathcal{A}}=J \mathfrak{H}_{z z}(t, \hbar) A,\left.\quad \mathcal{A}\right|_{t=s}=\mathbb{I}_{2 n \times 2 n}, \\
& \mathcal{A}(t, s)=\left(\begin{array}{cc}
\lambda_{4}^{\top}(t, s) & -\lambda_{2}^{\top}(t, s) \\
-\lambda_{3}^{\top}(t, s) & \lambda_{1}^{\top}(t, s)
\end{array}\right),
\end{aligned}
$$

and

$$
S(t, \hbar, \mathfrak{g}(t, \mathfrak{C}))=\int_{0}^{t}\{\langle\vec{P}(t, \hbar, \mathfrak{C}), \dot{\vec{X}}(t, \hbar, \mathfrak{C})\rangle-\mathfrak{H}(t, \hbar, \mathfrak{C})\} d t
$$

Then, the following theorem is true:

Theorem 2. Let an operator $\hat{U}_{\varkappa}(t, s, \cdot)$ act on a given function $\psi(\vec{x})$, taken at an initial time $s$, as follows:

$$
\hat{U}_{\varkappa}(t, s, \psi)(\vec{x})=\int_{\mathbb{R}^{n}} G_{\varkappa}\left(\vec{x}, \vec{y}, t, s, \mathfrak{g}(t, \mathfrak{C}(\psi)), \mathfrak{g}(s, \mathfrak{C}(\psi)) \psi(\vec{y}) d^{n} y .\right.
$$

Here $G_{\varkappa}(\vec{x}, \vec{y}, t, s, \mathfrak{g}(t, \mathfrak{C}(\psi), \mathfrak{g}(s, \mathfrak{C}(\psi))))$ is determined by (38), (41), and the parameters $\mathfrak{C}(\psi)$ are obtained from

$$
\left.\mathfrak{g}(t, \mathfrak{C})\right|_{t=s}=\mathfrak{g}_{0}(\psi)=\frac{1}{\|\psi\|^{2}}\langle\psi|\hat{\mathfrak{g}}| \psi\rangle
$$

Then, the function

$$
\Psi(\vec{x}, t)=\hat{U}_{\varkappa}(t, s, \psi)(\vec{x})
$$

is an exact solution of the Cauchy problem for equations (27), (28) with the initial condition $\left.\Psi(\vec{x}, t)\right|_{t=s}=\psi(\vec{x})$, and the operator $\hat{U}_{\varkappa}(t, s, \cdot)$ is the evolution operator for the GPE (27) that acts on the class of trajectory concentrated functions (7).

For the evolution operator (42), the following properties can be verified by direct computation.

Theorem 3. The operator $\hat{U}_{\varkappa}^{-1}(t, s, \cdot)$,

$$
\begin{aligned}
\hat{U}_{\varkappa}^{-1}(t, s, \psi)(\vec{x}) & =\int_{\mathbb{R}^{n}} G_{\varkappa}^{-1}(\vec{x}, \vec{y}, t, s, \mathfrak{g}(t,(\psi)), \mathfrak{g}(s, \mathfrak{C}(\psi))) \psi(\vec{y}) d^{n} y \\
& =\int_{\mathbb{R}^{n}} G_{\varkappa}(\vec{x}, \vec{y}, s, t, \mathfrak{g}(s, \mathfrak{C}(\psi)), \mathfrak{g}(t,(\psi))) \psi(\vec{y}) d^{n} y,
\end{aligned}
$$

is the left inverse operator to $\hat{U}_{\varkappa}(t, s, \cdot)$ of $(42)$, so that

$$
\hat{U}_{\varkappa}^{-1}\left(t, s, \hat{U}_{\varkappa}(t, s, \psi)\right)(\vec{x})=\psi(\vec{x}), \quad \psi \in \mathcal{P}_{\hbar}^{0} .
$$

Corollary 1. For a partial solution $\Psi(\vec{x}, t)$ of the GPE (27), we have

$$
\hat{U}_{\varkappa}\left(t, s, \hat{U}_{\varkappa}^{-1}(t, s, \Psi(t))\right)(\vec{x})=\Psi(\vec{x}, t) .
$$


Proof. Indeed, according to Theorem 2 we have $\Psi(\vec{x}, t)=\hat{U}_{\varkappa}(t, s, \psi)(\vec{x}), \psi(\vec{x})=\left.\Psi(\vec{x}, t)\right|_{t=s}$. In view of (46), the left-hand side of equation (47) can be written as

$$
\hat{U}_{\varkappa}\left(t, s, \hat{U}_{\varkappa}^{-1}(t, s, \Psi(t))\right)(\vec{x})=\hat{U}_{\varkappa}\left[t, s, \hat{U}_{\varkappa}^{-1}\left(t, s, \hat{U}_{\varkappa}(t, s, \psi)\right)\right](\vec{x})=\hat{U}_{\varkappa}(t, s, \psi)(\vec{x})=\Psi(\vec{x}, t) .
$$

Thus, the statement is proven.

Theorem 4. The operators $\hat{U}_{\varkappa}(t, \cdot)=\hat{U}_{\varkappa}(t, 0, \cdot)$ possess the group property

$$
\hat{U}_{\varkappa}(t+s, \psi)(x)=\hat{U}_{\varkappa}(t, \Psi(s))(\vec{x}), \quad \Psi(\vec{x}, s)=\hat{U}_{\varkappa}(s, \psi)(\vec{x}) .
$$

Proof. The functions $\Psi(\vec{x}, t)=\hat{U}_{\varkappa}(t+s, \psi)(\vec{x})$ and $\Psi(\vec{x}, t)$ of (44) are partial solutions of equation (31) with the same trajectory $\mathfrak{g}(t, \mathfrak{C})$ in the extended phase space, and (48) is valid for the evolution operator of the linear equation (31). Then, it is also valid for $\mathfrak{g}(t, \mathfrak{C}(\psi))$ corresponding to the nonlinear evolution operator (42).

Substituting $t+s \rightarrow t$ in (48), we find

$$
\begin{aligned}
& \hat{U}_{\varkappa}(t, \psi)(\vec{x})=\hat{U}_{\varkappa}(t-s, \Psi(s))(\vec{x}), \\
& \left.\Psi(\vec{x}, s)\right|_{s=0}=\psi(\vec{x})
\end{aligned}
$$

for a solution $\Psi(\vec{x}, t)$ of the Cauchy problem for the GPE (27) with the initial condition (50).

\section{Symmetry and nonlinear superposition}

\section{Symmetry operators}

A symmetry operator, by definition, maps a solution of an equation into another solution of this equation. Direct finding of symmetry operators for a given nonlinear equation is an intricate problem because of the nonlinearity of the determining equations.

It is rare for this problem to be solved (see, for example, [23]). The symmetry analysis of differential equations deals mainly with generators of one-parametric families of symmetry operators (symmetries of an equation) determined by linear equations [8, 9, 11, 12, 10]. Using the evolution operator $\hat{U}_{\varkappa}(t, s, \cdot)$ given by $(42)$, we can formulate a general form for symmetry operators of the Gross-Pitaevskii equation (27).

Let â be an operator acting in $\mathcal{P}_{\hbar}^{0},\left(\hat{a}: \mathcal{P}_{\hbar}^{0} \rightarrow \mathcal{P}_{\hbar}^{0}\right)$ and $\Psi(\vec{x}, t)$ is an arbitrary function of the $\mathcal{P}_{\hbar}^{t}$ class $\left(\Psi(\vec{x}, t) \in \mathcal{P}_{\hbar}^{t}\right)$. Consider an operator $\hat{\mathrm{A}}(\cdot)$, such that

$$
\Phi(\vec{x}, t)=\hat{\mathrm{A}}(\Psi(t))(\vec{x})=\hat{U}_{\varkappa}\left(t, \hat{\mathrm{a}} \hat{U}_{\varkappa}^{-1}(t, \Psi(t))\right)(\vec{x}) .
$$

If $\Psi(\vec{x}, t)$ is a solution of the GPE (27), then $\Phi(\vec{x}, t)$ is also a solution of equation (27). This follows immediately from Theorems 2 and 3 , and Corollary 1.

Thus, the operator $\hat{\mathrm{A}}(\cdot)$ determined by $(51)$ is a symmetry operator for the GPE (27).

Assume now that operator $\hat{b}$ and its operator exponent $\exp (\alpha \hat{\mathfrak{b}})$ act in the $\mathcal{P}_{\hbar}^{0}$ class, i.e., $\hat{\mathrm{b}}: \mathcal{P}_{\hbar}^{0} \rightarrow \mathcal{P}_{\hbar}^{0}$ and $\exp (\alpha \hat{\mathrm{b}}): \mathcal{P}_{\hbar}^{0} \rightarrow \mathcal{P}_{\hbar}^{0}$, where $\alpha$ is a parameter.

Define a one-parametric family of operators $\hat{\mathrm{B}}(\alpha, \cdot)$ via their action on an arbitrary function $\Psi(\vec{x}, t) \in \mathcal{P}_{\hbar}^{t}$ as

$$
\hat{\mathrm{B}}(\alpha, \Psi(t))(\vec{x})=\hat{U}_{\varkappa}\left(t, \exp \{\alpha \hat{\mathrm{b}}\} \hat{U}_{\varkappa}^{-1}(t, \Psi(t))\right)(\vec{x}) .
$$

By analogy with the aforesaid, the operators $\hat{\mathrm{B}}(\alpha, \cdot)$ constitute a one-parametric family of the symmetry operators of equation (27). 
It is easy to verify the group property

$$
\hat{\mathrm{B}}(\alpha+\beta, \Psi(t))(\vec{x})=\hat{\mathrm{B}}(\alpha, \hat{\mathrm{B}}(\beta, \Psi(t)))(\vec{x}), \quad \forall \Psi(\vec{x}, t) \in \mathcal{P}_{\hbar}^{t} .
$$

Differentiating (52) with respect to the parameter $\alpha$, we obtain for $\alpha=0$

$$
\hat{\mathrm{C}}(\Psi(t))(\vec{x})=\left.\frac{d}{d \alpha} \hat{\mathrm{B}}(\alpha, \Psi(t))(\vec{x})\right|_{\alpha=0}=\left.\frac{d}{d \alpha} \hat{U}_{\varkappa}\left(t, \exp \{\alpha \hat{\mathrm{b}}\} \hat{U}_{\varkappa}^{-1}(t, \Psi(t))\right)(\vec{x})\right|_{\alpha=0} .
$$

The operator $\hat{\mathrm{C}}(\cdot)$ determined by $(54)$ is a generator of the one-parametric family of symmetry operators $(52)$.

Note that the operator $\hat{\mathrm{C}}(\cdot)$ is not a symmetry operator for equation (27) since the parameters $\mathfrak{C}$ in the evolution operator $\hat{U}_{\varkappa}(t, \cdot)(42)$ depend on $\alpha$. Indeed, the parameters $(\mathfrak{C})$ found from equation (43)

$$
\left.\mathfrak{g}(t, \mathfrak{C})\right|_{t=0}=\langle\exp \{\alpha \hat{\mathrm{b}}\} \phi|\hat{\mathfrak{g}}| \exp \{\alpha \hat{\mathrm{b}}\} \phi\rangle, \quad \phi(\vec{x})=\hat{U}_{\varkappa}^{-1}(t, \Psi(t))(\vec{x}), \quad \Psi(\vec{x}, t) \in \mathcal{P}_{\hbar}^{t}
$$

include the parameter $\alpha$ explicitly. Therefore, equation (54) includes the derivatives of the evolution operator $\hat{U}_{\varkappa}(t, \cdot)$ with respect to the parameters $\mathfrak{C}$, and (54) is different in form from the symmetry operator (51).

\section{Nonlinear superposition}

The nonlinear superposition principle for the GPE (27) can be formulated in terms of the evolution operator

Let

$$
\Psi_{1}(\vec{x}, t)=\hat{U}_{\varkappa}\left(t, \psi_{1}\right)(\vec{x}), \quad \Psi_{2}(\vec{x}, t)=\hat{U}_{\varkappa}\left(t, \psi_{2}\right)(\vec{x}) \in \mathcal{P}_{\hbar}^{t}
$$

be two partial solutions to the GPE (27) corresponding to the initial functions $\psi_{1}(\vec{x}), \psi_{2}(\vec{x})$ $\in \mathcal{P}_{\hbar}^{0}$, respectively.

Then, the function

$$
\Psi(\vec{x}, t)=\hat{U}_{\varkappa}\left(t, c_{1} \psi_{1}+c_{2} \psi_{2}\right)(\vec{x})
$$

is a solution of equation (27) which corresponds to the initial function $c_{1} \psi_{1}(\vec{x})+c_{2} \psi_{2}(\vec{x})$, $c_{1}, c_{2} \in \mathbb{R}^{1}$. Therefore,

$$
\Psi(\vec{x}, t)=\hat{U}_{\varkappa}\left(t, c_{1} \hat{U}_{\varkappa}^{-1}\left(t, \Psi_{1}(t)\right)+c_{2} \hat{U}_{\varkappa}^{-1}\left(t, \Psi_{2}(t)\right)\right)(\vec{x})
$$

is a solution of equation (27) which corresponds to the solutions $\Psi_{1}(\vec{x}, t), \Psi_{2}(\vec{x}, t)$ of the form (55), and equation (56) is the superposition principle for the GPE (27).

\section{$5 \quad$ Examples}

\section{$3 \mathrm{D}$ case}

Consider equations (2) and (3) in a 3 -dimensional space with operators $\hat{\mathcal{H}}(t), \hat{V}(t, \Psi)$ of the form

$$
\begin{aligned}
& \hat{\mathcal{H}}(t)=\frac{1}{2 m}\left(\hat{\vec{p}}-\frac{e}{c} \vec{A}(\vec{x}, t)\right)^{2}-e\langle\vec{E}(t), \vec{x}\rangle+\frac{k}{2} \vec{x}^{2}, \\
& V(\hat{z}, \hat{w}, t)=V(\vec{x}-\vec{y})=V_{0}\left(1-\frac{1}{2 \gamma^{2}}(\vec{x}-\vec{y})^{2}\right) .
\end{aligned}
$$


The external field in the linear operator (57) is the superposition of a constant magnetic field $\vec{H}=(0,0, H)$ with vector potential $\vec{A}=\frac{1}{2} \vec{H} \times \vec{x}$, an electric field $\vec{E}(t)=(E \cos \omega t, E \sin \omega t, 0)$ periodic in time with frequency $\omega$, and the field of an isotropic oscillator with potential $\frac{k}{2} \vec{x}^{2}$, $k>0$. The operator $\hat{\mathcal{H}}(t)$ is the same as in [15], and $V(\vec{x}-\vec{y})$ is obtained from the Taylor expansion of the Gaussian potential $V(\vec{x}-\vec{y})=V_{0} \exp \left[-\frac{(\vec{x}-\vec{y})^{2}}{2 \gamma^{2}}\right]$. In notations (27)-(30), we have

$$
\begin{aligned}
& \mathcal{H}_{z z}=\left(\begin{array}{cc}
\mathcal{H}_{p p} & \mathcal{H}_{p x} \\
\mathcal{H}_{x p} & \mathcal{H}_{x x}
\end{array}\right), \quad \mathcal{H}_{p p}=\frac{1}{m} \mathbb{I}_{3 \times 3}, \quad \mathcal{H}_{x x}=\operatorname{diag}\left\{m \omega_{1}^{2}, m \omega_{1}^{2}, m \omega_{2}^{2}\right\} ; \\
& \mathcal{H}_{z}=\left(\overrightarrow{\mathcal{H}}_{p}, \overrightarrow{\mathcal{H}}_{x}\right)^{\top}, \quad \overrightarrow{\mathcal{H}}_{p}=0, \quad \overrightarrow{\mathcal{H}}_{x}=-e \vec{E}(t)
\end{aligned}
$$

the nonzero elements of the $3 \times 3$ matrix $\mathcal{H}_{p x}\left(=\mathcal{H}_{x p}^{\top}\right)$ are: $\mathcal{H}_{p_{1} x_{2}}=-\mathcal{H}_{p_{2} x_{1}}=\frac{1}{2} \omega_{H}$. Here, $\omega_{H}=\frac{e H}{m c}$ is a cyclotron frequency, $\omega_{n l}(v)^{2}=\frac{|\tilde{\varkappa} v|}{m}$ is a "nonlinear frequency", $\omega_{0}^{2}=\frac{k}{m}, \omega_{1}^{2}=$ $\omega_{0}^{2}+\left(\frac{\omega_{H}}{2}\right)^{2}-\zeta\left(V_{0}\right) \omega_{n l}(\eta)^{2}, \omega_{2}^{2}=\omega_{0}^{2}-\zeta\left(V_{0}\right) \omega_{n l}(\eta)^{2}, \zeta(a)=\operatorname{sign}(\tilde{\varkappa} a), a \in \mathbb{R}^{1}, \eta=\frac{V_{0}}{\gamma^{2}}$. The nonzero matrices in (6) are: $W_{x x}=W_{y y}=-W_{x y}=-\frac{1}{\gamma^{2}} V_{0} \mathbb{I}_{3 \times 3}$.

For the block matrix $\mathcal{A}(t)$ of the form (40), the matrices $\lambda_{1}, \lambda_{2}, \lambda_{3}, \lambda_{4}$ are of order $3 \times 3$ and they can be written as

$$
\lambda_{1}=\lambda_{4}=\frac{d \hat{\mathbf{r}}(t)}{d t} \hat{\mathbf{u}}(t), \quad \lambda_{2}=-m \frac{d^{2} \hat{\mathbf{r}}(t)}{d t^{2}} \hat{\mathbf{u}}(t), \quad \lambda_{3}=-\frac{1}{m} \hat{\mathbf{r}}(t) \hat{\mathbf{u}}(t),
$$

where

$$
\hat{\mathbf{r}}(t)=\operatorname{diag}\left\{\frac{\sin \left(\omega_{1} t\right)}{\omega_{1}}, \frac{\sin \left(\omega_{1} t\right)}{\omega_{1}}, \frac{\sin \left(\omega_{2} t\right)}{\omega_{2}}\right\}, \quad \hat{\mathbf{u}}(t)=\left(\begin{array}{lll}
\cos \left(\frac{1}{2} \omega_{H} t\right) & -\sin \left(\frac{1}{2} \omega_{H} t\right) & 0 \\
\sin \left(\frac{1}{2} \omega_{H} t\right) & \cos \left(\frac{1}{2} \omega_{H} t\right) & 0 \\
0 & 0 & 1
\end{array}\right)
$$

Here $\hat{\mathbf{u}}(t)$ is an $S O(3)$ matrix, $\hat{\mathbf{u}}^{\boldsymbol{\top}}(t) \hat{\mathbf{u}}(t)=\hat{\mathbf{u}}(t) \hat{\mathbf{u}}^{\boldsymbol{\top}}(t)=\mathbb{I}_{3 \times 3}$. Denote by $(\vec{P}(t, \hbar, \mathfrak{C}), \vec{X}(t, \hbar, \mathfrak{C}))$ the general solution of the system (16) with (59), for which we use for short the notation $(\vec{P}(t), \vec{X}(t))$. We use similar notation for $(41), S(t, \hbar, \mathfrak{g}(t, \mathfrak{C}))=S(t)$.

Let us introduce the functions

$$
\begin{aligned}
\mathcal{G}( & \left.\Delta x, \Delta y, t, s, P(t), P(s), \omega, \omega_{H}, \mathfrak{C}\right)=\sqrt{\frac{m \omega}{2 i \pi \hbar \sin (\omega(t-s))}} \exp \left\{\frac{i}{\hbar}(P(t) \Delta x-P(s) \Delta y)\right\} \\
& \times \exp \left\{\frac{i \omega m}{2 \hbar \sin (\omega(t-s))}\left(\cos (\omega(t-s))\left(\Delta x^{2}+\Delta y^{2}\right)-2 \cos \left(\frac{\omega_{H}}{2}(t-s)\right) \Delta x \Delta y\right)\right\}
\end{aligned}
$$

and

$$
\mathcal{G}(\Delta x, \Delta y, t, s, P(t), P(s), \omega, \mathfrak{C})=\left.\mathcal{G}\left(\Delta x, \Delta y, t, s, P(t), P(s), \omega, \omega_{H}, \mathfrak{C}\right)\right|_{\omega_{H}=0}
$$

Then, the Green function (38) reads

$$
\begin{aligned}
& G_{\varkappa}(\vec{x}, \vec{y}, t, s, \mathfrak{g}(t, \mathfrak{C}), \mathfrak{g}(s, \mathfrak{C}))=\mathcal{G}\left(\Delta x_{1}, \Delta y_{1}, t, s, P_{1}(t), P_{1}(s), \omega_{1}, \omega_{H}, \mathfrak{C}\right) \\
& \quad \times \mathcal{G}\left(\Delta x_{2}, \Delta y_{2}, t, s, P_{2}(t), P_{2}(s), \omega_{1}, \omega_{H}, \mathfrak{C}\right) \mathcal{G}\left(\Delta x_{3}, \Delta y_{3}, t, s, P_{3}(t), P_{3}(s), \omega_{2}, \mathfrak{C}\right) \\
& \quad \times \exp \left\{\frac{i}{\hbar}[S(t)-S(s)]\right\} \exp \left\{\frac{i}{\hbar}\left(-m \omega_{1} \frac{\sin \left(\frac{\omega_{H}}{2}(t-s)\right)}{\sin \left(\omega_{1}(t-s)\right)}\left(\Delta x_{1} \Delta y_{2}-\Delta x_{2} \Delta y_{1}\right)\right)\right\} .
\end{aligned}
$$

The nonlinear evolution operator (42) is constructed with the use of (62) in which $\mathfrak{C}$ are determined by (43). 


\section{D case}

To demonstrate symmetry operators in explicit form consider the one-dimensional case of the GPE (27) following [16].

The operators (57) and (58) for $n=1$ take the form

$$
\mathcal{H}(\hat{z}, t)=\frac{\hat{p}^{2}}{2 m}+\frac{k x^{2}}{2}-e E x \cos \omega t, \quad V(\hat{z}, \hat{w}, t)=\frac{1}{2}\left[a x^{2}+2 b x y+c y^{2}\right] .
$$

Here, $\vec{x}=x \in \mathbb{R}^{1}, \vec{y}=y \in \mathbb{R}^{1} ; k>0, m, e, E, a, b$, and $c$ are parameters of the potential.

In notations $(27)-(30)$, we have $\mathfrak{H}(t, \hbar, \mathfrak{g}(t, \mathfrak{C}))=\frac{1}{2 m} P^{2}(t, \mathfrak{C})+\frac{1}{2} k X^{2}(t, \mathfrak{C})-e E X(t, \mathfrak{C}) \cos \omega t+$ $\frac{\tilde{\varkappa}}{2} c \sigma_{x x}(t, \mathfrak{C})+\frac{\tilde{\varkappa}}{2}(a+2 b+c) X^{2}(t, \mathfrak{C})$,

$$
\left.\mathfrak{H}_{z}(t, \mathfrak{g}(t, \mathfrak{C}))=\left(\begin{array}{c}
\frac{1}{m} P(t, \mathfrak{C}) \\
m \tilde{\Omega}^{2} X(t, \mathfrak{C})-e E \cos \omega t
\end{array}\right), \quad \mathfrak{H}_{z z}(t, \mathfrak{g}(t, \mathfrak{C}))\right)=\left(\begin{array}{cc}
\frac{1}{m} & 0 \\
0 & m \Omega^{2}
\end{array}\right) .
$$

The Green function of the linear associated Schrödinger equation (31) follows directly from (60) and (62). It reads

$$
G_{\varkappa}(x, y, t, s, \mathfrak{g}(t, \mathfrak{C}), \mathfrak{g}(s, \mathfrak{C}))=\mathcal{G}(\Delta x, \Delta y, t, s, P(t), P(s), \Omega, \mathfrak{C}) \exp \left\{\frac{i}{\hbar}[S(t)-S(s)]\right\} .
$$

Here, $\omega_{0}=\sqrt{k / m}$ and $\Omega^{2}=\omega_{0}^{2}+\zeta(a) \omega_{n l}^{2}(a)$. The evolution operator $\hat{U}_{\varkappa}(t, s, \cdot)(42)$ is determined by (64), where the constants $\mathfrak{C}$ are changed by $\mathfrak{C}(\psi)$ determined by equation $(23): \mathfrak{g}(s, \mathfrak{C})=$ $\mathfrak{g}(\mathfrak{C})=\frac{1}{\|\psi\|^{2}}\langle\psi|\hat{\mathfrak{g}}| \psi\rangle, \mathfrak{g}(\mathfrak{C})=\left(P(\mathfrak{C}), X(\mathfrak{C}), \sigma_{p p}(\mathfrak{C}), \sigma_{p x}(\mathfrak{C}), \sigma_{x x}(\mathfrak{C})\right)$. The LASE (31), (32) is known to have a partial solution in Gaussian form. For the operators (63), this solution can be obtained as

$$
\Phi_{0}^{(0)}(x, t, \mathfrak{g}(t, \mathfrak{C}))=\sqrt[4]{\frac{m \Omega}{\pi \hbar}} e^{-i \Omega t / 2} \exp \left\{\frac{i}{\hbar}(S(t, \hbar, \mathfrak{g}(t, \mathfrak{C}))+P(t, \mathfrak{C}) \Delta x)-\frac{m \Omega}{2 \hbar} \Delta x^{2}\right\}
$$

Setting $\psi(x)=\Phi_{0}^{(0)}(x, 0, \mathfrak{g}(0, \mathfrak{C})) \equiv \Phi_{0}^{(0)}(x, 0)$ in $(23)$, we can find the parameters $\mathfrak{C}$ in the form $\mathfrak{C}(\psi)=\mathfrak{C}_{0}=\mathfrak{C}\left(\Phi_{0}^{(0)}(0)\right)=\left(\frac{p_{0}}{m \tilde{\Omega}}, x_{0}, 0,0, \frac{\hbar}{2 m \Omega}\right)^{\top}$, where $\tilde{\Omega}^{2}=\omega_{0}^{2}+\zeta(a+b) \omega_{n l}^{2}(a+b)$, $p_{0}=\left\langle\Phi_{0}^{(0)}|\hat{p}| \Phi_{0}^{(0)}\right\rangle /\left\|\Phi_{0}^{(0)}\right\|^{2}$, and $x_{0}=\left\langle\Phi_{0}^{(0)}|x| \Phi_{0}^{(0)}\right\rangle /\left\|\Phi_{0}^{(0)}\right\|^{2}$. Then,

$$
\Psi_{0}^{(0)}\left(x, t, \mathfrak{C}_{0}\right)=\Phi_{0}^{(0)}(x, t, \mathfrak{g}(t, \mathfrak{C}(\psi)))
$$

is a partial solution of the 1-dimensional GPE (27).

Let us change the operators $\hat{a}$ in relation (51) by the operators $\hat{a}^{+}$and $\hat{a}$ of the form

$$
\hat{a}=\frac{1}{\sqrt{2 \hbar m \Omega}}\left[\Delta \hat{p}_{0}-i m \Omega \Delta x_{0}\right], \quad \hat{a}^{+}=\frac{1}{\sqrt{2 \hbar m \Omega}}\left[\Delta \hat{p}_{0}+i m \Omega \Delta x_{0}\right],
$$

where $\Delta \hat{p}_{0}=-i \hbar \partial_{x}-p_{0}$ and $\Delta x_{0}=x-x_{0}$. Then, the operators $\widehat{A}^{( \pm)}(\cdot)$ determined by the relations

$$
\widehat{A}^{(+)}(\Psi(t))(x)=\widehat{U}_{\varkappa}\left(t, \hat{a}^{+} \widehat{U}_{\varkappa}^{-1}(t, \Psi(t))\right)(x), \quad \widehat{A}^{(-)}(\Psi(t))(x)=\widehat{U}_{\varkappa}\left(t, \hat{a} \widehat{U}_{\varkappa}^{-1}(t, \Psi(t))\right)(x),
$$

where $\Psi(x, t) \in \mathcal{P}_{\hbar}^{t}$, are the symmetry operators of equation $(27)$. With the operators $\widehat{A}^{( \pm)}(\cdot)$, we obtain the set of solutions for the GPE

$$
\Psi_{n}^{(0)}\left(x, t, \mathfrak{g}\left(t, \mathfrak{C}_{n}\right)\right)=\frac{1}{\sqrt{n !}}\left(\widehat{A}^{(+)}(\cdot)\right)^{n} \Psi_{0}^{(0)}\left(x, t, \mathfrak{g}\left(t, \mathfrak{C}_{0}\right)\right)
$$




$$
=\frac{i^{n}}{\sqrt{n !}} e^{-i n \Omega t}\left(\frac{1}{\sqrt{2}}\right)^{n} H_{n}\left(\sqrt{\frac{m \Omega}{\hbar}} \Delta x\right) \Psi_{0}^{(0)}\left(x, t, \mathfrak{g}\left(t, \mathfrak{C}_{n}\right)\right)
$$

where $n \in \mathbb{Z}^{+}, H_{n}(\xi)$ are Hermite polynomials and

$$
\mathfrak{C}_{n}^{T}(\psi)=\left(0, \frac{e E}{m\left(\tilde{\Omega}^{2}-\omega^{2}\right)}, 0,0, \frac{\hbar(2 n+1)}{2 m \Omega}\right)^{\top} .
$$

It can be verified that

$$
\begin{aligned}
& \widehat{A}^{(+)}\left(\Psi_{n}^{(0)}\left(t, \mathfrak{g}\left(t, \mathfrak{C}_{n}\right)\right)\right)(x)=\sqrt{n+1} \Psi_{n+1}^{(0)}\left(x, t, \mathfrak{g}\left(t, \mathfrak{C}_{n+1}\right)\right), \\
& \widehat{A}^{(-)}\left(\Psi_{n}^{(0)}\left(t, \mathfrak{g}\left(t, \mathfrak{C}_{n}\right)\right)\right)(x)=\sqrt{n} \Psi_{n-1}^{(0)}\left(x, t, \mathfrak{g}\left(t, \mathfrak{C}_{n-1}\right)\right)
\end{aligned}
$$

and the operators $\widehat{A}^{( \pm)}(\cdot)$ are nonlinear analogs of "creation-annihilation" operators. It can be noted that the functions $\Psi_{n}^{(0)}\left(x, t, \mathfrak{g}\left(t, \mathfrak{C}_{n}\right)\right)$ satisfy the quasi-periodic condition

$$
\Psi_{n}^{(0)}\left(x, t+T, \mathfrak{C}_{n}^{T}\right)=e^{-i \mathcal{E}_{n} T / \hbar} \Psi_{n}^{(0)}\left(x, t, \mathfrak{C}_{n}^{T}\right),
$$

where $\mathcal{E}_{n}$ is the quasi-energy. The spectrum of quasi-energies is given by the relation

$$
\begin{aligned}
\mathcal{E}_{n}= & -\frac{e^{2} E^{2}}{2 m\left(\tilde{\Omega}^{2}-\omega^{2}\right)}-\frac{e^{2} E^{2}\left[\omega^{2}-\omega_{0}^{2}-\zeta(a+2 b+c) \omega_{\mathrm{nl}}^{2}(a+2 b+c)\right]}{4 m\left(\tilde{\Omega}^{2}-\omega^{2}\right)^{2}} \\
& +\hbar\left(\Omega+\frac{\tilde{\varkappa} c}{2 m \Omega}\right)\left(n+\frac{1}{2}\right) .
\end{aligned}
$$

\section{Concluding remarks}

The evolution operator (42) obtained in Section 3 in explicit form enables one to look in a new fashion at the problem of construction of semiclassically concentrated solutions to the nonlocal Gross-Pitaevskii equation (2). In particular, the semiclassical asymptotics constructed in [13, 14] can be presented in more compact and visual form. In addition, the evolution operator leads to the nonlinear superposition principle (56) and to the general form of symmetry operators (51). The latter can be used for study of symmetries of the Gross-Pitaevskii equation under consideration as long as direct finding of the symmetries by means of solution of the determining equations is a nontrivial problem.

\section{Acknowledgements}

The work was supported by the President of the Russian Federation, Grant No NSh-1743.2003.2.

[1] Cornell E.A., Wieman C.E., Nobel lecture: Bose-Einstein condensation in a dilute gas, the first 70 years some recent experiments, Rev. Mod. Phys., 2002, V.74, 875-893.

Ketterle W., Nobel lecture: When atoms behave as waves: Bose-Einstein condensation and the atom laser, Rev. Mod. Phys., 2002, V.74, 1131-1151.

[2] Pitaevskii L.P., Vortex lines in an imperfect Bose gas, Zh. Eksper. Teor. Fiz., 1961, V.40, $646-651$ (in Russian).

[3] Gross E.P., Structure of a quantized vortex in boson systems, Nuovo Cimento, 1961, V.20, N 3, $454-477$.

[4] Kivshar Y.S., Pelinovsky D.E., Self-focusing and transverse instabilities of solitary waves, Phys. Rep., 2000, V.331, N 4, 117-195.

[5] Bang O., Krolikowski W., Wyller J., Rasmussen J.J., Collapse arrest and soliton stabilization in nonlocal nonlinear media, nlin.PS/0201036.

[6] Zakharov V.E., Shabat A.B., Exact theory of two-dimensional self-focusing and one-dimensional selfmodulation of waves in nonlinear media, Zh. Eksper. Teor. Fiz., 1971, V.61, 118-134 (English transl.: Sov. Phys. JETP, 1971, V.34, 62-69). 
[7] Zakharov V.E., Manakov S.V., Novikov S.P., Pitaevsky L.P., Theory of solitons: The inverse scattering method, Moscow, Nauka, 1980 (English transl.: New York, Plenum, 1984).

[8] Ovsjannikov L.V., Group analysis of differential equations, Moscow, Nauka, 1978 (English transl.: New York, Academic Press, 1982).

[9] Anderson R. L., Ibragimov N.H., Lie-Bäcklund transformations in applications, Philadelphia, SIAM, 1979.

[10] Olver P.J., Application of Lie groups to differential equations, New York, Springer, 1986.

[11] Fushchich W.I., Shtelen W.M., Serov N.I., Symmetry analysis and exact solutions of equations of nonlinear mathematical physics, Dordrecht, Kluwer, 1993.

[12] Fushchich W.I., Nikitin A.G., Symmetries of equations of quantum mechanics, New York, Allerton Press Inc., 1994.

[13] Belov V.V., Trifonov A.Yu., Shapovalov A.V., The trajectory-coherent approximation and the system of moments for the Hartree type equation, Int. J. Math. and Math. Sci., 2002, V.32, N 6, 325-370.

[14] Belov V.V., Trifonov A.Yu., Shapovalov A.V., Semiclassical trajectory-coherent approximation for the Hartree type equation, Teor. Mat. Fiz., 2002, V.130, N 3, 460-492 (English transl.: Theor. Math. Phys., 2002, V.130, N 3, 391-418).

[15] Shapovalov A.V., Trifonov A.Yu., Lisok A.L., Semiclassical approach to the geometric phase theory for the Hartree type equation, in Proceedinds of Fifth International Conference "Symmetry in Nonlinear Mathematical Physics" (June 23-29, 2003, Kyiv), Editors A.G. Nikitin, V.M. Boyko, R.O. Popovych and I.A. Yehorchenko, Proceedings of Institute of Mathematics, Kyiv, 2004, V.50, Part 3, 1454-1465.

[16] Lisok A.L., Trifonov A.Yu., Shapovalov A.V., The evolution operator of the Hartree-type equation with a quadratic potential, J. Phys. A: Math. Gen., 2004, V.37, 4535-4556.

[17] Karasev M.V., Maslov V.P., Nonlinear Poisson brackets: geometry and quantization, Moscow, Nauka, 1991 (English transl.: Nonlinear Poisson brackets: geometry and quantization, Ser. Translations of Mathematical Monographs, V.119, Providence, RI, Amer. Math. Soc., 1993).

[18] Maslov V.P., The complex WKB method for nonlinear equations, Moscow, Nauka, 1977 (English transl.: The complex WKB method for nonlinear equations. I. Linear theory, Basel - Boston - Berlin, Birkhauser Verlag, 1994).

[19] Belov V.V., Dobrokhotov S.Yu., Semiclassical Maslov asymptotics with complex phases. I. General approach, Teor. Mat. Fiz., 1992, V.92, N 2, 215-254 (English transl.: Theor. Math. Phys., 1992, V.92, N 2, 843-868).

[20] Ehrenfest P., Bemerkung über die angenherte Gültigkeit der klassishen Mechanik innerhalb der Quanten Mechanik, Zeits. Phys., 1927, Bd.45, 455-457.

[21] Malkin M.A., Manko V.I., Dynamic symmetries and coherent states of quantum systems, Nauka, Moscow, 1979 (in Russian).

[22] Perelomov A.M., Generalized coherent states and their application, Berlin, Springer-Verlag, 1986.

[23] Meirmanov A.M., Pukhnachov V.V., Shmarev S.I., Evolution equations and Lagrangian coordinates, New York - Berlin, Walter de Gruyter, 1994. 